\title{
EFEITO DOS GASES DE PROTEÇÃO NA MICROESTRUTURA E NAS CINÉTICAS DE OXIDAÇÃO A ALTAS TEMPERATURAS AO AR DE JUNTAS SOLDADAS DE UM AÇO INOXIDÁVEL AUSTENÍTICO ATRAVÉS DO PROCESSO MIG/MAG
}

André de Albuquerque Vicente Débora Arruda Cabral ${ }^{2}$ Denise Crocce Romano Espinosa ' Jorge Alberto Soares Tenório '

\section{Resumo}

Foram estudadas as microestruturas e as cinéticas de oxidação a altas temperaturas ao ar de quatro juntas soldadas de um aço inoxidável austenítico produzidas através do processo MIG/MAG com diferentes gases de proteção. Utilizou-se o metal de adição 22. 12. HT e diferentes gases de proteção: $100 \% \mathrm{Ar}, \mathrm{Ar}+2 \% \mathrm{CO}_{2}, \mathrm{Ar}+4 \% \mathrm{CO}_{2}$ e $\mathrm{Ar}+20 \% \mathrm{CO}_{2}$. Para cada mistura gasosa, foi soldado um corpo de prova. Estudou-se a variação das frações volumétricas de ferrita delta em função dos diferentes gases de proteção utilizados. Observou-se aumento no teor de carbono e consequente diminuição das frações volumétricas de ferrita delta, diminuições significativas nos teores de $\mathrm{Cr}$, $\mathrm{Si}$ e $\mathrm{Mn}$ em relação à composição química do consumível de soldagem e consequente diminuição da resistência à oxidação a altas temperaturas ao ar dos metais depositados com gases de proteção com maiores concentrações de $\mathrm{CO}_{2}$.

Palavras-chave: Aços inoxidáveis; Gases de proteção; Fração volumétrica; Oxidação a altas temperaturas; MIG/MAG.

\section{EFFECT OF SHIELDING GAS IN MICROSTRUCTURE AND KINECTICS OF HIGH TEMPERATURE OXIDATION IN AIR OF WELDED JOINTS OF AN AUSTENITIC STAINLESS STEEL THROUGH THE MIG/MAG PROCESS}

\begin{abstract}
The microstructures and the high temperature oxidation kinectics in air of four welded joints of austenitic stainless steel produced by the MIG/MAG process with different shielding gases were studied. Were used the welding wire 22. I2. $\mathrm{HT}$, and different shielding gases: $100 \% \mathrm{Ar}, \mathrm{Ar}+2 \% \mathrm{CO}_{2}, \mathrm{Ar}+4 \% \mathrm{CO}_{2}$ and $\mathrm{Ar}+20 \% \mathrm{CO}_{2}$. For each gas mixture was welded a specimen. The variation of the volume fractions of delta ferrite according to the different shielding gases used, were studied. There was an increase in carbon content and decreasing of volume fraction of delta ferrite, a decrease in $\mathrm{Cr}$, Si and $\mathrm{Mn}$ content when compared with the chemical composition of the welding wire decreasing the high temperature oxidation resistance in air of weld metals deposited with shielding gases with higher concentrations of $\mathrm{CO}_{2}$. Keywords: Stainless steels; Shielding gases; Volume fraction; High temperature oxidation; MIG/MAG.
\end{abstract}

\section{INTRODUÇÃO}

Além do ferro, cromo e níquel, os aços inoxidáveis têm em sua composição outros elementos químicos que podem estabilizar a ferrita e a austenita. Levando em conta os seus efeitos ferritizantes e austenitizantes, Schaeffler [I] agrupou estes elementos em duas expressões denominadas de cromo equivalente e níquel equivalente, respectivamente e propôs um diagrama que é apresentado na Figura I. O diagrama de Schaeffler não é um diagrama de equilíbrio. Ele foi determinado experimentalmente com o objetivo de prever-se o teor de ferrita delta em soldas de aços inoxidáveis.

Além das expressões de cromo equivalente (Creq $=\% \mathrm{Cr}+\% \mathrm{Mo}+\mathrm{I}, 5 \% \mathrm{Si}+0,5 \% \mathrm{Nb})$ e de níquel

'Departamento de Engenharia Química, Escola Politécnica, Universidade de São Paulo - USP, São Paulo, SP, Brasil. E-mail: andre.vicente@usp.br

${ }^{2}$ Departamento de Engenharia Metalúrgica e de Materiais, Escola Politécnica, Universidade de São Paulo - USP, São Paulo, SP, Brasil. 


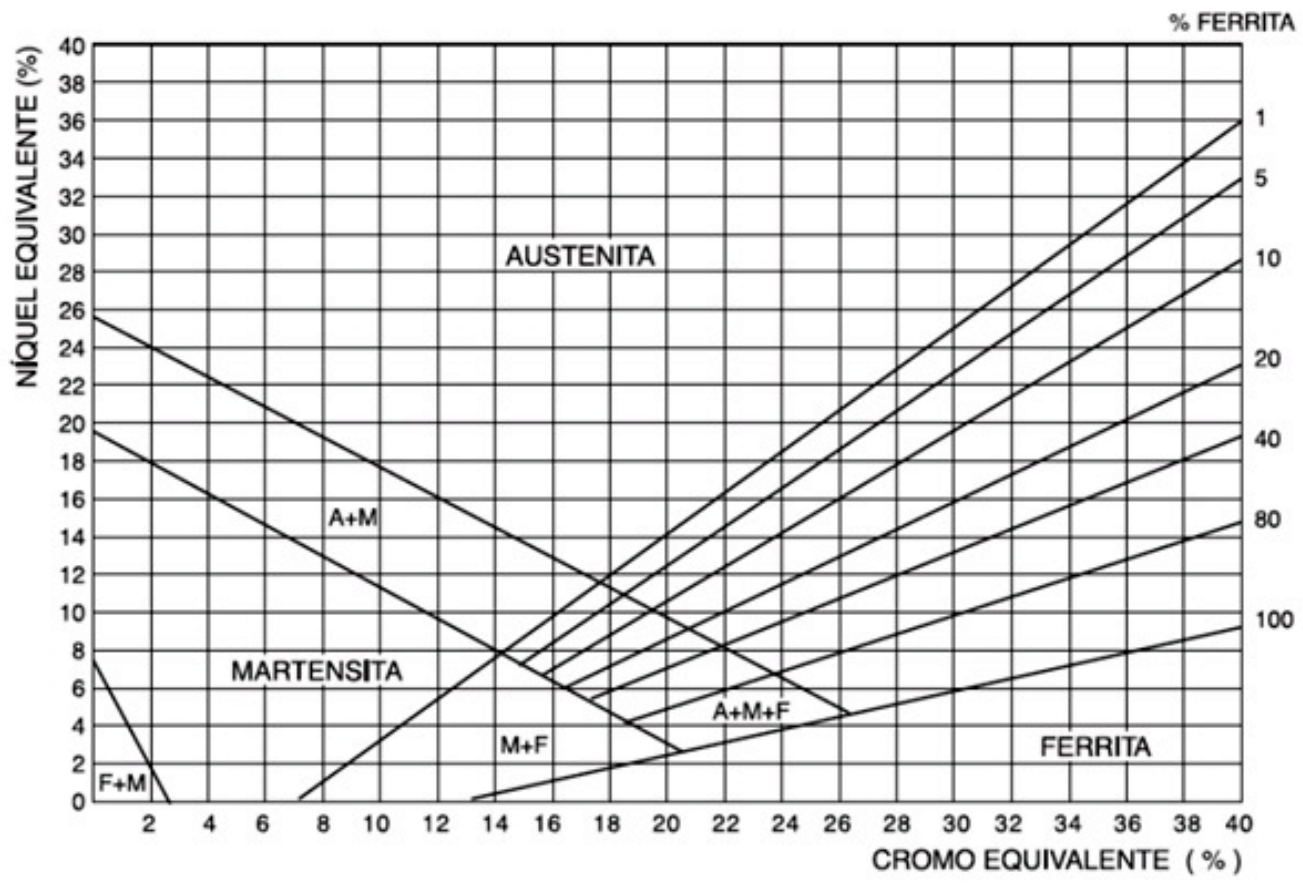

Figura I. Diagrama de Schaeffler [I].

equivalente $(\mathrm{Nieq}=\% \mathrm{Ni}+0,5 \% \mathrm{Mn}+30 \% \mathrm{C})$ propostas por Schaeffler, foram sugeridas na literatura várias outras expressões incorporando outros elementos, utilizando outras condições de solidificação e diferentes composições químicas [2-8].

A primeira alteração marcante foi feita por Delong [9], que inclui o efeito austenitizante do nitrogênio. Dependendo das condições de solidificação, os fatores para os elementos nas expressões de cromo e níquel podem variar bastante e alguns elementos que não influem nas expressões, dependendo do processo, podem ser importantes ao tratar-se de diferentes modos de solidificação.

Tomando-se como exemplo um aço inoxidável austenítico do tipo AISI $3 \mid 6$, o qual solidifica através de uma solidificação ferrítica-austenítica, O. Hammar e U. Svensson [6] mostraram que a adição de carbono e nitrogênio no aço diminui a fração de ferrita $\delta$ primária até chegar ao ponto em que se formava austenita primária. Existe, portanto, um valor de carbono equivalente que pode mudar o modo de solidificação deste aço. A Figura 2 mostra a variação da fração de ferrita primária em função do carbono equivalente segundo a fórmula $\mathrm{Ceq}=\% \mathrm{C}+0,65 \% \mathrm{~N}$ em peso.

Assim, O. Hammar e Svensson [6] propuseram uma nova relação para valores de cromo e níquel equivalentes colocando o efeito do nitrogênio e carbono para o níquel e o efeito do titânio para o cromo. Afim de prever o modo de solidificação dos aços inoxidáveis através das expressões de cromo e niquel equivalentes propostas por $\mathrm{O}$. Hammar e Svensson $[6,10]$, Suutala e Moisio [II] rearranjaram os resultados obtidos em diversos trabalhos $[5,7,12-16]$ com diferentes velocidades de solidificação. Quando a relação $\mathrm{Creq} /$ Nieq $<I, 5$ a solidificação poderá ser

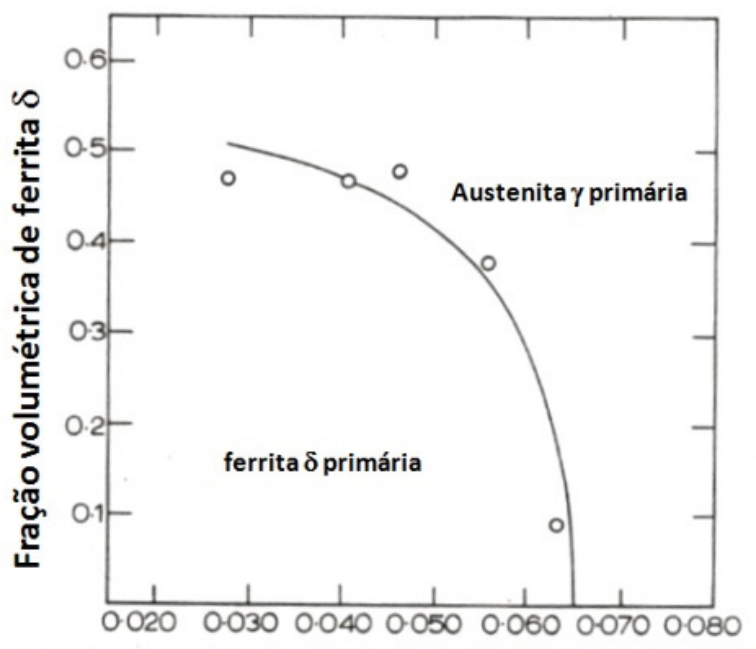

Carbono equivalente $(C+0,65 \mathrm{~N})(\%$ em massa)

Figura 2. Fração de ferrita primária em função do $C_{\text {eq }}[6]$.

austenítica (modo I) ou austenítica-ferrítica (modo II). Quando a relação $1,5<\mathrm{Creq} / \mathrm{Nieq}<2,0$ a solidificação será ferrítica-austenítica (modo III). E, finalmente, quando Creq/Nieq $>2,0$ a solidificação será ferrítica (modo IV).

A Tabela I apresenta as expressões de cromo e níquel equivalentes sugeridas por diferentes pesquisadores [1,6,9-10,17-19]:

Quando um metal é exposto a um gás oxidante a temperatura elevada, pode ocorrer corrosão por reação direta com o gás sem a necessidade da presença de um eletrólito líquido. A velocidade da reação aumenta substancialmente 
Tabela I. Expressões de cromo e níquel equivalentes [3]

\begin{tabular}{|c|c|c|c|c|c|c|c|c|}
\hline Autor & & Schaeffler & DeLong & $\begin{array}{c}\text { Pryce e } \\
\text { Andrews (I) }\end{array}$ & Giraldeng & $\begin{array}{c}\text { Potak e } \\
\text { Sagalevich } \\
\text { apud Hull } \\
\end{array}$ & Hull (2) & $\begin{array}{c}\text { Hammar e } \\
\text { Svensson }\end{array}$ \\
\hline Condições & & Soldagem & $\begin{array}{c}\text { Soldagem a } \\
\text { Quente }\end{array}$ & Laminados & Fundidos & Fundidos & $\begin{array}{c}\text { Fundidos } \\
\text { em } \\
\text { Coquilha }\end{array}$ & $\begin{array}{l}\text { Análises } \\
\text { Térmicas }\end{array}$ \\
\hline \multirow{9}{*}{$\begin{array}{l}\text { Fatores para } \\
\text { Constantes } \\
\text { dos Elementos } \\
\text { Ferritizantes }\end{array}$} & $\mathrm{Cr}$ & 1,00 & 1,00 & 1,00 & 1,00 & 1,00 & 1,00 & 1,00 \\
\hline & Mo & $\mathrm{I}, 00$ & $\mathrm{I}, 00$ & $\mathrm{I}, 00$ & 2,00 & 1,00 & $\mathrm{I}, 2 \mathrm{I}$ & $\mathrm{I}, 37$ \\
\hline & Si & 1,50 & 1,50 & 3,00 & 1,50 & 2,00 & 0,48 & 1,50 \\
\hline & $\mathbf{N b}$ & 0,50 & 0,50 & $4 \mathrm{Nb}^{\prime}$ & - & 0,90 & 0,14 & 2,00 \\
\hline & Ti & - & - & $10 \mathrm{Ti}$ & 4,00 & 4,00 & 2,20 & 3,00 \\
\hline & $\mathbf{w}$ & - & - & - & - & 0,50 & 0,72 & - \\
\hline & $\mathbf{v}$ & - & - & - & - & 1,50 & 2,27 & - \\
\hline & Al & - & - & - & 3,00 & 4,00 & 2,48 & - \\
\hline & $\mathbf{T a}$ & - & - & - & - & - & 0,21 & - \\
\hline \multirow{6}{*}{$\begin{array}{l}\text { Fatores para } \\
\text { Constantes } \\
\text { dos Elementos } \\
\text { Austenitizantes }\end{array}$} & $\mathbf{N i}$ & 1,00 & 1,00 & 1,00 & $\mathrm{I}, 00$ & $\mathrm{I}, 00$ & 1,00 & 1,00 \\
\hline & $M n$ & 0,50 & 0,50 & 0,50 & - & 0,50 & $M n^{\prime}$ & 0,31 \\
\hline & C & 30,00 & 30,00 & 21,00 & 30,00 & 27,00 & 24,50 & 22,00 \\
\hline & $\mathbf{N}$ & - & 30,00 & 11,50 & 20,00 & 27,00 & 18,40 & 14,20 \\
\hline & $\mathrm{Cu}$ & - & - & 0,44 & - & 0,33 & 0,44 & 1,00 \\
\hline & Co & - & - & - & - & 0,40 & $0,4 \mathrm{I}$ & - \\
\hline
\end{tabular}

(I) $\mathrm{Nb}^{\prime}=\mathrm{Nb}-8[(\mathrm{C}-0,03)+\mathrm{N}], \mathrm{Ti}^{\prime}=\mathrm{Ti}-4[(\mathrm{C}-0,03)+\mathrm{N}] ;$ (2) Mn' $=0, \mathrm{II} \mathrm{Mn}-0,0086 \mathrm{Mn}^{2}$

com a temperatura. A película superficial normalmente aumenta sua espessura como resultado da reação na interface película/gás ou metal/película devido ao transporte de cátions e ânions através da película, que se comporta como um eletrólito sólido. Para a formação de películas contínuas e não porosas o transporte iônico através da película é a etapa controladora do processo. A estabilidade termodinâmica e certas características morfológicas da película formada são fatores chave na determinação da resistência de uma determinada liga a um meio específico. $O$ crescimento inicial da película é normalmente bastante rápido [20].

Se a película for sólida, não porosa e cobrir completamente a superfície do metal, a velocidade de reação irá diminuir quando a espessura da película atingir alguns milhares de angstrons conforme o transporte de massa através da camada se tornar a etapa controladora do processo. A subsequente taxa de oxidação depende dos detalhes do mecanismo de transporte, que pode ser por potencial ou gradientes de concentração ou por migração por caminhos preferenciais. Onde a difusão for a etapa controladora do processo, a cinética normalmente segue uma lei parabólica na qual a velocidade da reação cai progressivamente com o tempo [20]. A Figura 3 apresenta as possíveis cinéticas de crescimento da película [2I].

Quando vários óxidos se formam (por exemplo, $\mathrm{Fe}_{2} \mathrm{O}_{3}, \mathrm{Fe}_{3} \mathrm{O}_{4}$ e $\mathrm{FeO}$ ), os mesmos terão pressões parciais de dissociação diferentes e o óxido que for mais rico em oxigênio se dissociará antes no óxido menos rico e somente depois no metal [22].

$\mathrm{Na}$ oxidação de ligas, a formação seletiva de um especificado óxido é governada pela reação preferencial dos

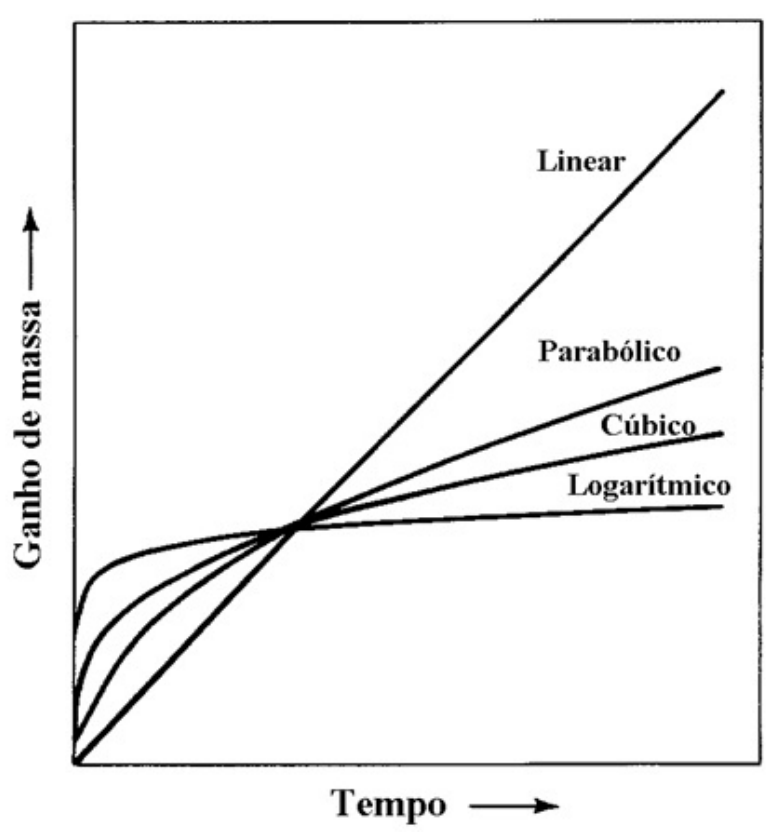

Figura 3. Cinéticas de oxidação (ganho de massa $X$ tempo) [2I].

elementos de liga que formam o composto termodinamicamente mais estável.

\section{I.2 Objetivos do Trabalho}

O presente trabalho tem como objetivo a caracterização microestrutural e o levantamento das cinéticas de oxidação a altas temperaturas ao ar de quatro juntas soldadas de aço inoxidável austenítico produzidas através do processo 
MIG/MAG com diferentes gases de proteção, dentro de um projeto que visava estudar o comportamento de juntas soldadas de aços inoxidáveis austeníticos a altas temperaturas.

\section{MATERIAIS E MÉTODOS}

Para a soldagem dos corpos de prova utilizou-se o processo MIG/MAG. Foram utilizados o consumível 22. 12. $\mathrm{HT}$ e diferentes concentrações de gases de proteção: $100 \%$ $\mathrm{Ar}, \mathrm{Ar}+2 \% \mathrm{CO}_{2}, \mathrm{Ar}+4 \% \mathrm{CO}_{2}$ e $80 \% \mathrm{Ar}+20 \% \mathrm{CO}_{2}$. Para cada mistura gasosa, foi soldado um corpo de prova. A Tabela 2 apresenta a composição química, fornecida pela fabricante, do metal de adição (22.12.HT) utilizado neste trabalho:

Ajustou-se o equipamento MIG/MAG de forma a permitir uma soldagem estável para os quatro gases de proteção. A Figura 4 descreve como os corpos de prova foram confeccionados:

Para a confecção dos corpos de prova, utilizou-se como metal base chapas de aço inoxidável austenítico AISI 304L com $6 \mathrm{~mm}$ de espessura, cortadas nas dimensões $70 \times 50 \mathrm{~mm}$. O dimensional dos corpos de prova conforme a Figura 4 foi:

- Comprimento do depósito: $>50 \mathrm{~mm}$

- Altura do depósito: $>20 \mathrm{~mm}$

- Largura do depósito: $>30 \mathrm{~mm}$

- Quantidade de camadas depositadas: $>5$

Utilizou-se MIG/MAG convencional. A Tabela 3 apresenta os parâmetros de soldagem utilizados na confecção dos corpos de prova soldados:

A Figura 5 apresenta os corpos de prova soldados:

Para as análise metalográficas e ensaios de oxidação a altas temperaturas ao ar objetivou-se as regiões centrais dos CPs cortados. A Figura 6 ilustra a região central cortada de um CP soldado para a retirada das amostras para a caracterização metalográfica e confecção de corpos de prova para os ensaios de oxidação a altas temperaturas ao ar. Os corpos de prova de foram usinados a $20 \mathrm{~mm}$ do metal base para todas as juntas soldadas.
Empregou-se o lixamento manual convencional utilizando-se lixas d'água ( $180,220,320,400$ e 600 mesh) seguido de polimento com alumina até superfície especular. As amostras foram atacadas através de ataque eletrolítico com $\mathrm{NaOH} 20 \%$ com 6,5 V por 2,5 minutos. Todas as amostras foram analisadas em um microscópio óptico com 100 X de aumento. Realizaram-se medições das frações volumétricas de ferrita e austenita em todos os corpos de prova sempre a $20 \mathrm{~mm}$ do metal base. No intuito de quantificar as frações

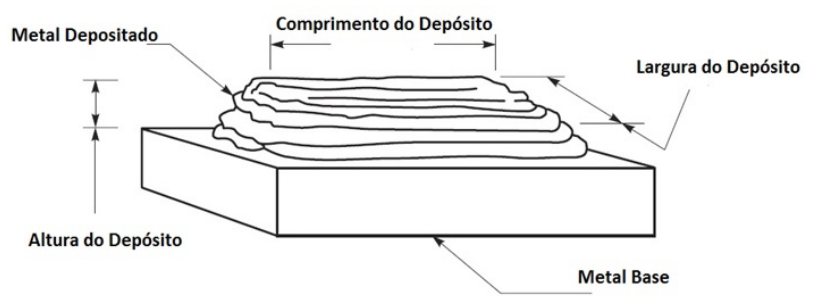

Figura 4. Esquema da confecção dos corpos de prova soldados através de processo de soldagem MIG/MAG.

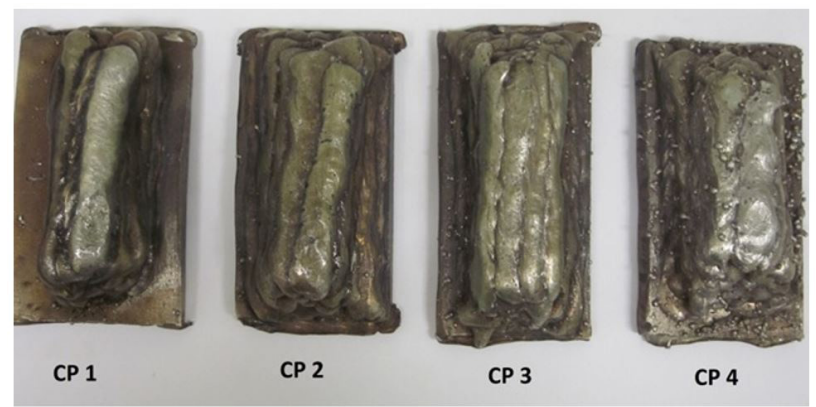

(a)

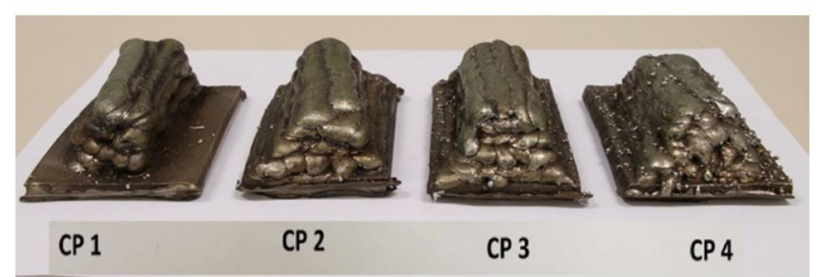

(b)

Figura 5. Fotos do CPs soldados. a) Vista superior b) Vista frontal. Os gases de proteção utilizados são: $\mathrm{CPI}: 100 \% \mathrm{Ar}, \mathrm{CP} 2 \mathrm{Ar}+2 \%$ $\mathrm{CO}_{2}, \mathrm{CP} 3: \mathrm{Ar}+4 \% \mathrm{CO}_{2}$ e CP4: $\mathrm{Ar}+20 \% \mathrm{CO}_{2}$.

Tabela 2. Composição química do metal de adição de soldagem [\% em massa]

\begin{tabular}{cccccccccc}
\hline Grau & $\mathbf{C}$ & $\mathbf{S i}$ & $\mathbf{M n}$ & $\mathbf{P}$ & $\mathbf{S}$ & $\mathbf{C r}$ & $\mathbf{N i}$ & $\mathbf{N}$ & $\mathbf{C e}$ \\
\hline 22.12.HT & 0,068 & 1,58 & 0,52 & 0,016 & 0,000 & 20,90 & 10,17 & 0,17 & 0,03 \\
\hline
\end{tabular}

Tabela 3. Parâmetros de soldagem utilizados na confecção dos corpos de prova soldados

\begin{tabular}{ccccccc}
\hline $\begin{array}{c}\text { Corpo de } \\
\text { Prova }\end{array}$ & $\begin{array}{c}\text { Gás de } \\
\text { Proteção }\end{array}$ & Vazão (I/min) & Corrente (A) & Tensão (V) & $\begin{array}{c}\text { Velocidade de Soldagem } \\
(\mathbf{m m} / \mathbf{m i n})\end{array}$ & $\begin{array}{c}\text { Energia de Soldagem } \\
(\mathbf{k J} / \mathbf{m m})\end{array}$ \\
\hline CPI & $\mathrm{Ar}$ & 20 & 204 & 26 & 240 & 1,33 \\
CP2 & $\mathrm{Ar}+2 \% \mathrm{CO}_{2}$ & 20 & 210 & 27 & 256 & 1,33 \\
CP3 & $\mathrm{Ar}+4 \% \mathrm{CO}_{2}$ & 20 & 212 & 26 & 253 & 1,31 \\
CP4 & $\mathrm{Ar}+20 \% \mathrm{CO}_{2}$ & 20 & 209 & 27 & 263 & 1,29 \\
\hline
\end{tabular}


volumétricas de ferrita utilizou-se o software analisador de imagens "Image J". Analisaram-se 10 micrografias de cada corpo de prova nos sentidos transversal e longitudinal conforme proposto pela norma ASTM E 562-02 [23].

Realizaram-se análises químicas em todos os corpos de prova sempre a $20 \mathrm{~mm}$ do metal base através de espectrômetro de emissão ótica, conforme a norma ASTM E 1086-08 [24].

As espessuras e dimensões dos corpos de prova para os ensaios de oxidação a altas temperaturas ao ar foram determinadas com o auxílio de um micrômetro. Através destas medições pode-se padronizar os ensaios de oxidação e obter-se os valores de ganho de massa por unidade de área. Antes do início dos ensaios de oxidação, todas as amostras

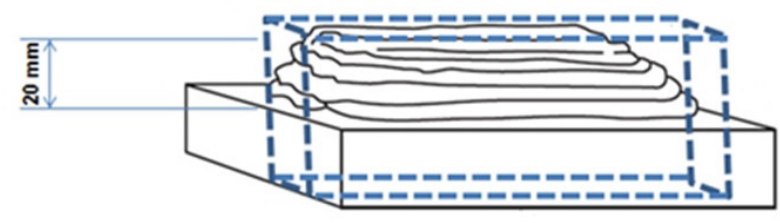

Figura 6. Região central de um CP soldado para caracterização metalográfica.

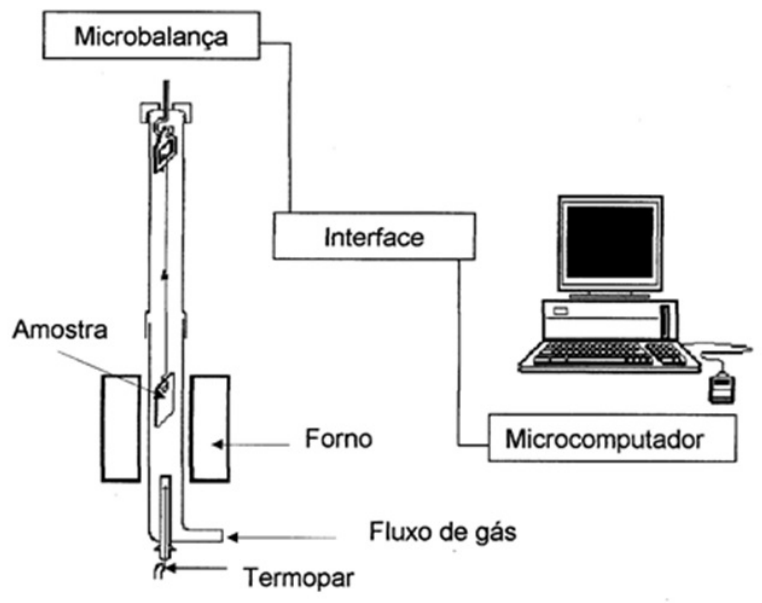

Figura 7. Esquema de funcionamento de uma balança termogravimétrica.
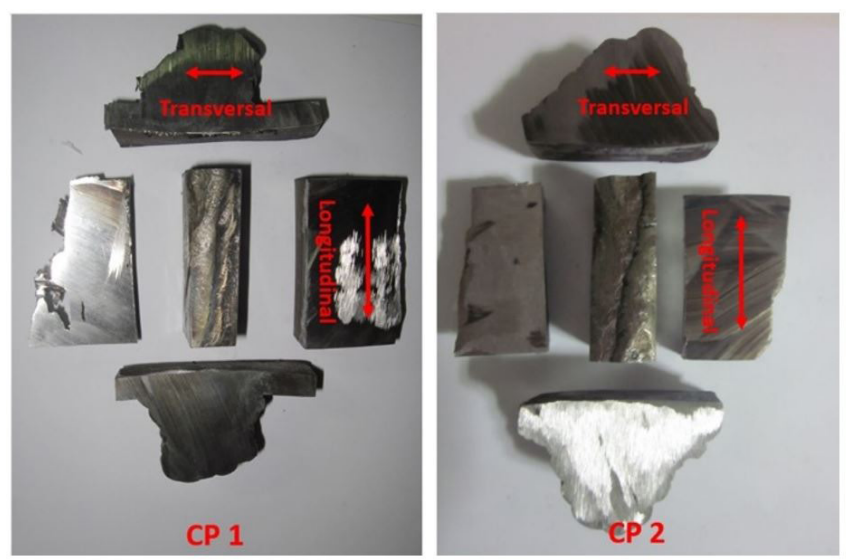

foram limpas com álcool e, posteriormente, com acetona em aparelho de ultrassom. Esta etapa da preparação é muito importante pois é uma forma de padronizar-se o acabamento superficial das amostras retirando resíduos de fluido de corte e/ou das lixas. Os ensaios de oxidação foram realizados utilizando-se balança termogravimétrica da marca Setaram do LAT-LAREX USP. Foram realizados ensaios isotérmicos ao ar nas temperaturas $800,900,1000$ e $1100^{\circ} \mathrm{C}$. Todos os ensaios foram realizados ao ar. $O$ tempo de duração dos ensaios é de 24 horas.

A Figura 7 apresenta o esquema de funcionamento de uma balança termogravimétrica.

\section{RESULTADOS E DISCUSSÃO}

A Figura 8 apresenta os corpos de prova soldados cortados indicando as regiões das análise microestruturais na transversal e na longitudinal dos mesmos:

As Figuras 9 e 10 apresentam micrografias obtidas a aproximadamente $20 \mathrm{~mm}$ do metal base nos sentidos transversal e longitudinal dos corpos de prova soldados, respectivamente.

Conforme esperado, a análise metalográfica revelou microestrutura austenítica-ferrítica. A análise metalográfica dos corpos de prova soldados revelou que o aumento da concentração de $\mathrm{CO}_{2}$ nos gases de proteção diminui a fração volumétrica de ferrita $\delta$ no metal depositado.

A constatação é evidenciada na Tabela 4 que apresenta as frações volumétricas de ferrita $\delta$ dos corpos de prova soldados nos sentidos transversal e longitudinal.

Utilizando-se as expressões propostas por DeLong para cromo e níquel equivalentes [9] é possível calcular as frações volumétricas de ferrita $\delta$ teóricas esperadas. A Tabela 5 apresenta as composições químicas do metal de adição utilizado e dos corpos de prova soldados, bem como os valores de cromo e níquel equivalentes utilizando-se as expressões de DeLong [9] e os valores de carbono equivalente utilizando-se a expressão de $\mathrm{O}$. Hammar e U. Svensson [6].
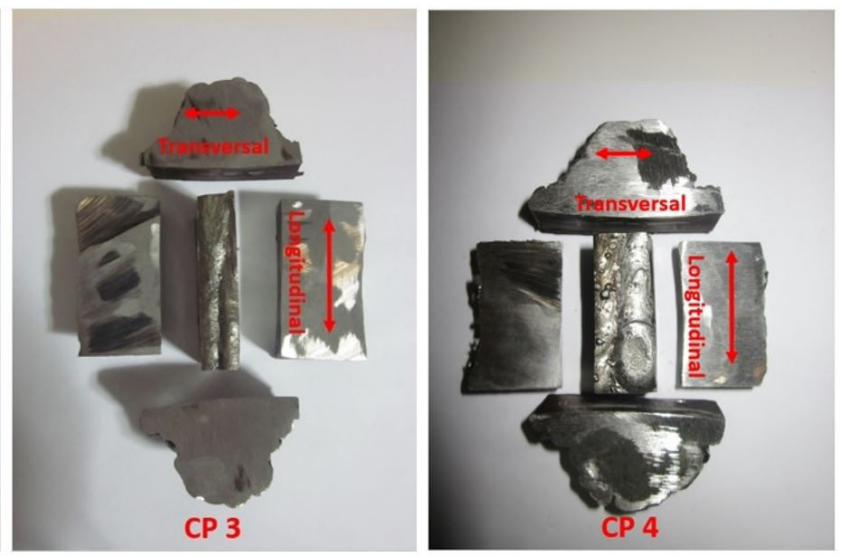

Figura 8. Regiões alvo dos CPs soldados para as análises microestruturais na transversal e longitudinal. 

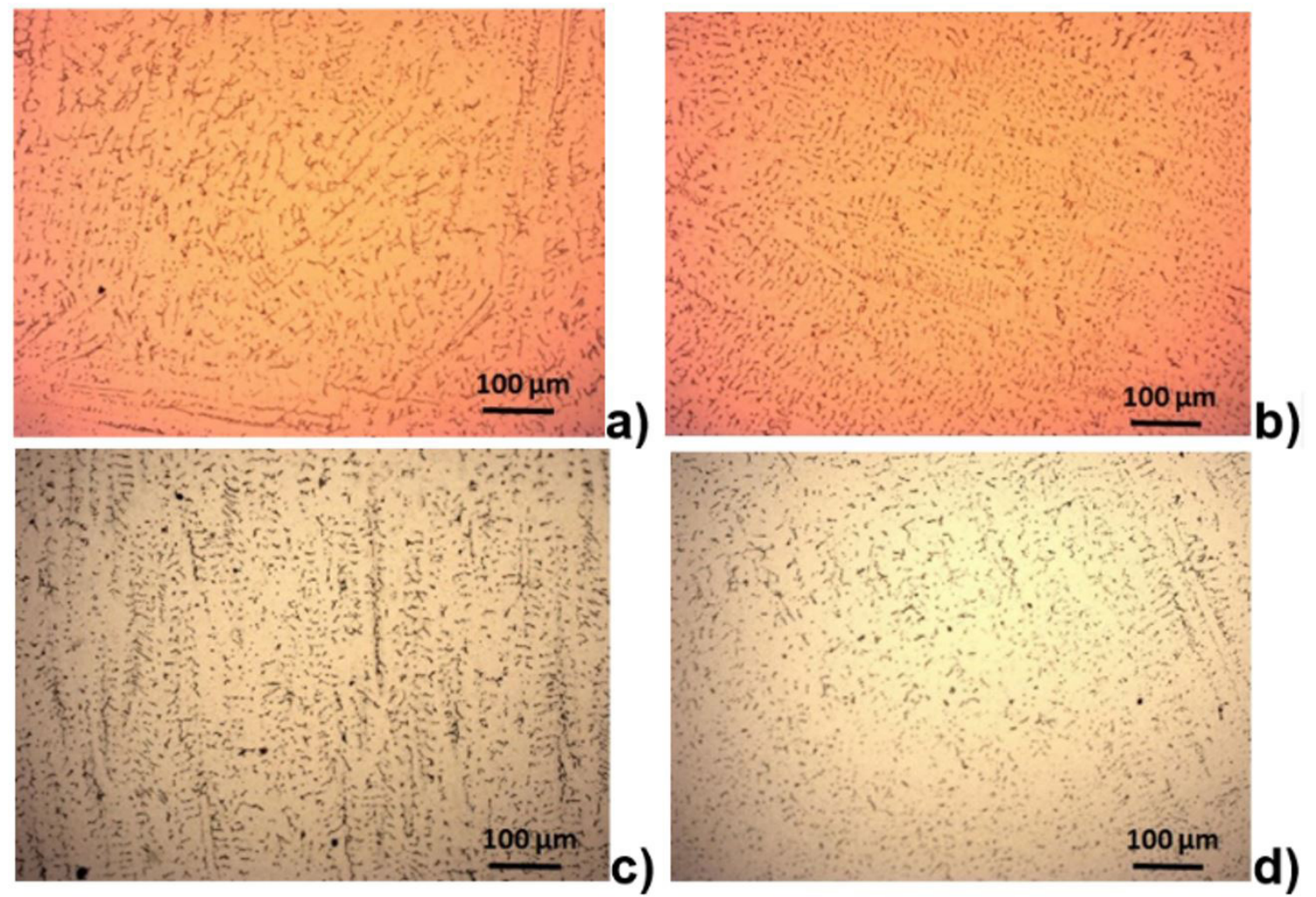

Figura 9. Micrografias dos cortes transversais dos corpos de prova soldados. a) CPI, b) CP2, c) CP3 e d) CP4 (ataque eletrolítico com $\mathrm{NaOH}$ $20 \%$ com $6,5 \vee$ por 2,5 minutos).
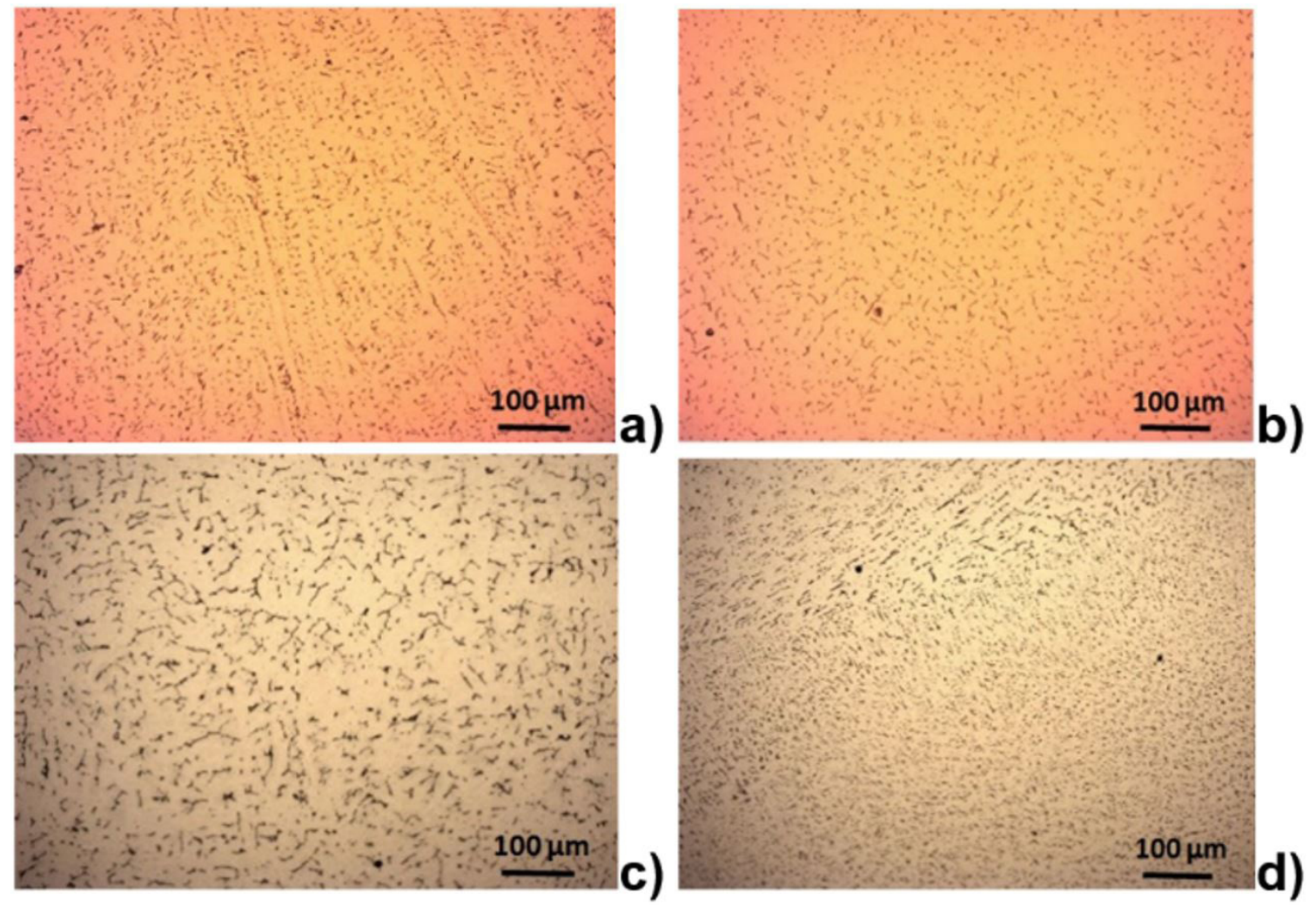

Figura 10. Micrografias dos cortes longitudinais dos corpos de prova soldados. a) CPI, b) CP2, c) CP3 e d) CP4 (ataque eletrolítico com $\mathrm{NaOH} 20 \%$ com $6,5 \mathrm{~V}$ por 2,5 minutos). 
$\mathrm{O}$ aumento da concentração de $\mathrm{CO}_{2}$ nos gases de proteção aumenta $\circ \mathrm{Ni}_{\mathrm{eq}}$ da liga através do aumento da concentração de $C$ no metal depositado, apesar da oxidação seletiva do $\mathrm{Mn}$. Os resultados obtidos sugerem que com aumento da concentração de $\mathrm{CO}_{2}$ nos gases de proteção ocorra uma diminuição no $\mathrm{Cr}_{\mathrm{eq}}$ das ligas devido à oxidação seletiva dos elementos $\mathrm{Cr}$ e Si. Quando a relação $\mathrm{Cr}_{\text {eq }} / \mathrm{Ni}_{\text {eq }}<\mathrm{I}, 5$ a solidificação poderá ser austenítica (modo l) ou austenítica-ferrítica (modo II). Todas as ligas estudadas apresentaram relação $\mathrm{Cr}_{\mathrm{eq}} / \mathrm{Ni}_{\mathrm{eq}}<\mathrm{I}, 5$. Os resultados obtidos sugerem que a solidificação das ligas estudadas seja austenítica-ferrítica (modo II).

Verifica-se que o aumento da concentração de $\mathrm{CO}_{2}$ nos gases de proteção ocorre diminuição do $\mathrm{Cr}_{\mathrm{eq}}$ e aumento do $\mathrm{Ni}_{\text {eq }}$, resultando na diminuição da relação $\mathrm{Cr}_{\text {eq }} / \mathrm{Ni}_{\text {eq }}$ das ligas.

A Figura I I apresenta as curvas de ganho de massa em função do tempo, obtidas para a amostra do aço inoxidável austenítico laminado $\left(253 \mathrm{MA}^{\circledR}\right)$ e para os 4 corpos de

Tabela 4. Fração Volumétrica de Ferrita $\delta$ dos Corpos de Prova Soldados

\begin{tabular}{|c|c|c|c|c|c|c|c|c|c|c|c|}
\hline $\begin{array}{c}\text { Fração Volumétrica } \\
\text { de Ferrita } \delta\end{array}$ & $\mathbf{I}$ & 2 & 3 & 4 & 5 & 6 & 7 & 8 & 9 & 10 & \\
\hline CPI Transversal & 8,0 & 7,6 & 7,7 & 7,3 & 6,4 & 9,4 & 8,2 & 10,1 & 8,9 & 10,3 & $8,4+/-1,2$ \\
\hline CPI Longitudinal & 7,6 & 8,4 & 7,4 & 7,3 & 6,5 & 6,8 & 6,2 & 5,0 & 5,3 & 7,5 & $6,9+/-1,1$ \\
\hline CPI Média & \multicolumn{11}{|c|}{$7,6+/-1,4$} \\
\hline CP2 Transversal & 7,3 & 7,9 & 8,5 & 7,3 & 8,4 & 8,7 & 10,8 & 7,0 & 6,5 & 7,5 & $8,0+/-1,1$ \\
\hline CP2 Longitudinal & 4,6 & 6,2 & 5,5 & 7,3 & 7,5 & 6,5 & 6,3 & 6,2 & 6,6 & 6,0 & $6,1+/-0,7$ \\
\hline CP2 Média & \multicolumn{11}{|c|}{$7,1+/-1,3$} \\
\hline CP3 Transversal & 6,7 & 5,8 & 6,0 & 7,3 & 4,8 & 5,3 & 7,5 & 6,5 & 7,4 & 8,2 & $6,3+/-1,2$ \\
\hline CP3 Longitudinal & 5,0 & 5,3 & 4,3 & 7,3 & 4,0 & 5,6 & 5,5 & 5,8 & 5,7 & 6,3 & $5,2+/-0,6$ \\
\hline CP3 Média & \multicolumn{11}{|c|}{$6,0+/-1,1$} \\
\hline CP4 Transversal & 4,4 & 4,3 & 3,7 & 7,3 & 2,7 & 3,3 & 4,1 & 3,8 & 3,1 & 3,3 & $3,6+/-0,5$ \\
\hline CP4 Longitudinal & 4,2 & 4,7 & 3,7 & 7,3 & 4,1 & 4,5 & 4,9 & 4,4 & 6,1 & 4,4 & $4,5+/-0,6$ \\
\hline CP4 Média & \multicolumn{11}{|c|}{$4,4+/-1,2$} \\
\hline
\end{tabular}

Tabela 5, Composições químicas do metal de adição 22, I2,HT e dos corpos de prova soldados

\begin{tabular}{|c|c|c|c|c|c|c|c|c|c|c|c|c|c|}
\hline & C & $\mathbf{S i}$ & $M n$ & $\mathbf{P}$ & $\mathbf{S}$ & $\mathrm{Cr}$ & $\mathbf{N i}$ & $\mathbf{N}$ & $\mathrm{Ce}$ & $\mathrm{Cr}_{\mathrm{eq}}$ & $\mathbf{N i}_{\text {eq }}$ & $\mathrm{Cr} / \mathrm{Ni}$ & $\mathbf{C}_{\mathrm{eq}}$ \\
\hline 253MA & 0,072 & 1,58 & 0,62 & 0,023 & 0,000 & 20,92 & 10,84 & 0,160 & 0,05 & 23,29 & $|8| 1 \mid$, & 1,29 & 0,176 \\
\hline $22,12, \mathrm{HT}$ & 0,068 & $\mathbf{I}, 58$ & 0,52 & 0,016 & 0,000 & 20,90 & 10,17 & 0,170 & 0,03 & 23,27 & 17,57 & 1,32 & 0,179 \\
\hline CPI & 0,059 & $|, 5|$ & 0,50 & 0,016 & 0,000 & 20,12 & $|0,3|$ & 0,172 & 0,03 & 22,39 & 17,49 & 1,28 & $0,17 \mid$ \\
\hline CP2 & 0,069 & $I, 45$ & 0,48 & 0,016 & 0,000 & 20,10 & 10,33 & 0,166 & 0,03 & 22,28 & 17,62 & 1,26 & 0,177 \\
\hline CP3 & 0,072 & 1,38 & 0,46 & 0,016 & 0,000 & 20,10 & 10,32 & 0,162 & 0,03 & 22,17 & 17,57 & 1,26 & 0,177 \\
\hline CP4 & 0,093 & 1,36 & 0,45 & 0,016 & 0,000 & 20,04 & 10,33 & 0,162 & 0,03 & 22,08 & $|8,2|$ & $I, 2 I$ & 0,198 \\
\hline
\end{tabular}

Creq=\%Cr+\%Mo+ I,5\%Si+0,5\%Nb [9]; Nieq=\%Ni+0,5\%Mn+30\%C+30\%N [9]; Ceq=\%C+0,65\%N [6].

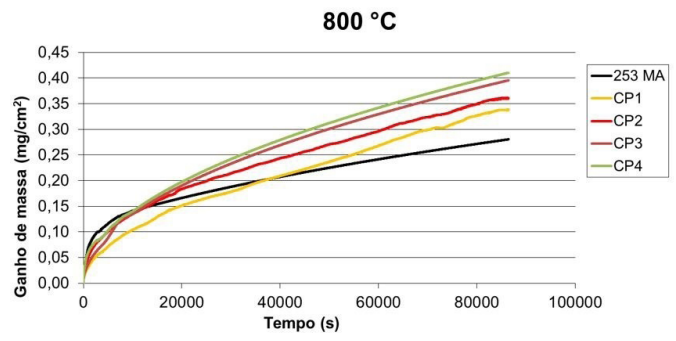

$1000{ }^{\circ} \mathrm{C}$

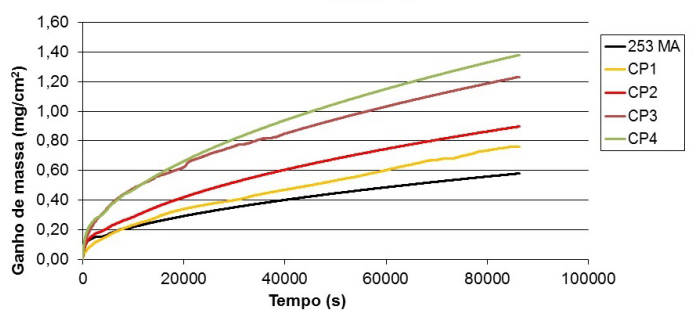

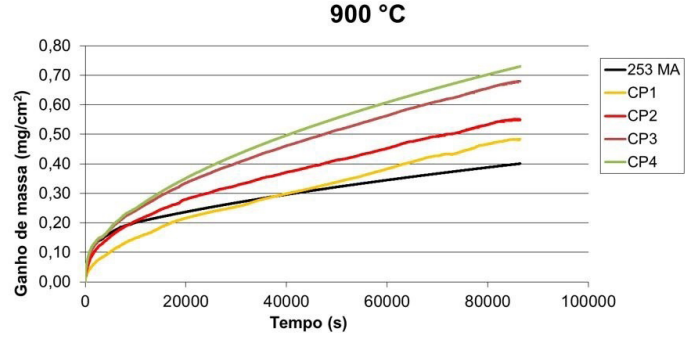

$1100^{\circ} \mathrm{C}$

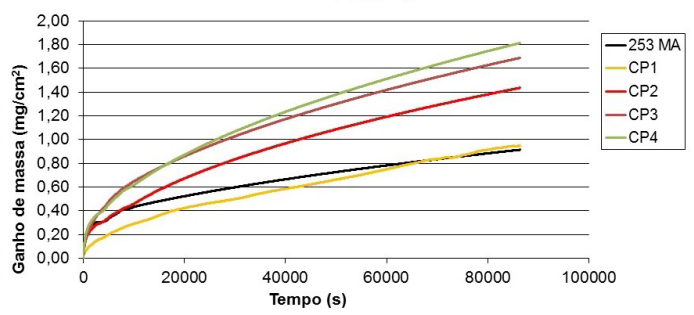

Figura II. Ensaios de oxidação ao ar por 24 horas a $800,900,1000$ e $1100^{\circ} \mathrm{C}$. 
prova soldados, nos ensaios isotérmicos de oxidação ao ar por 24 horas nas temperaturas $800,900,1000$ e $1100{ }^{\circ} \mathrm{C}$, respectivamente.

Observa-se, para as 5 ligas estudadas, o aumento das cinéticas de oxidação com o aumento da temperatura de ensaio de 800 até $1100^{\circ} \mathrm{C}$.

Foram testados os comportamentos parabólico, exponencial e linear para as cinéticas de crescimento dos óxidos formados.

A análise dos ensaios termogravimétricos do aço inoxidável austenítico laminado $\left(253 \mathrm{MA}^{\circledR}\right)$ bem como das 4 juntas soldadas, mostrou uma cinética de oxidação a altas temperaturas complexa, passando de logarítmica nos instantes iniciais para um posterior comportamento parabólico.

\section{CONCLUSÃO}

$\mathrm{O}$ aumento da concentração de $\mathrm{CO}_{2}$ nos gases de proteção aumenta $\circ \mathrm{Ni}_{\text {eq }}$ da liga através do aumento da concentração de $\mathrm{C}$ no metal depositado.
Os resultados obtidos sugerem que com aumento da concentração de $\mathrm{CO}_{2}$ nos gases de proteção ocorra uma diminuição no $\mathrm{Cr}_{\text {eq }}$ das ligas devido à oxidação seletiva dos elementos $\mathrm{Cr}$ e $\mathrm{Si}$.

O aumento da concentração de $\mathrm{C}$ diminui a fração volumétrica de ferrita $\delta$ no metal depositado.

Quando a relação $\mathrm{Cr}_{\text {eq }} / \mathrm{Ni}_{\text {eq }}<\mathrm{I}, 5$ a solidificação poderá ser austenítica (modo I) ou austenítica-ferrítica (modo II). Todas as ligas estudadas apresentaram relação $\mathrm{Cr}_{\text {eq }} / \mathrm{Ni}_{\text {eq }}<\mathrm{I}, 5$.

Os resultados obtidos sugerem que a solidificação das ligas estudadas seja austenítica-ferrítica (modo II).

Verifica-se que com o aumento da concentração de $\mathrm{CO}_{2}$ nos gases de proteção ocorre diminuição do $\mathrm{Cr}_{\text {eq }}$ e aumento do $\mathrm{Ni}_{\text {eq }}$, no metal depositado, resultando na diminuiçãa da relação $\mathrm{Cr}_{\text {eq }} / \mathrm{Ni}_{\text {eq }}$ das ligas.

$\mathrm{O}$ aumento da concentração de $\mathrm{CO}_{2}$ nos gases de proteção resulta em juntas soldadas menos resistentes à corrosão a altas temperaturas quando comparadas com o metal base 253MA devido à diminuição do Creq.

\section{REFERÊNCIAS}

I Schaeffler AL. Constitution diagram for stainless steel weld metal. Metal Progress. 1949;56(5):680-680B.

2 Padilha AF. Algumas observações sobre o descobrimento e o desenvolvimento dos aços inoxidáveis. In: Associação Brasileira de Metalurgia, Materiais e Mineração. Anais do I Seminário Brasileiro Sobre Aços Inoxidáveis; I989; São Paulo, Brasil. São Paulo: ABM; 1989. p. 3-I5.

3 Gazire RC. Efeito da Velocidade de Resfriamento na Microestrutura de solidificação de Aço Inoxidável Austenítico Tipo AISI 316L com Adições de Boro [dissertação]. São Paulo: Escola Politécnica da USP, Departamento de Engenharia Metalúrgica; 1993.

4 Kou S. Welding metallurgy. 2nd ed. Hoboken: John Wiley \& Sons Inc.; 2003. Post-solidification phase transformations; p. 21 6-242.

5 Fredriksson $\mathrm{H}$. The solidification sequence in a 18-8 stainless steel, investigated by directional solidification. Metallurgical Transactions. 1972;3:2989-2997.

6 Hammar O, Svensson U. Solidification and casting metals. London: Metals Society; 1979. Influence of steel composition on segregation and microstructure during solidification of austenitic stainless steels; p. 40I-4I0.

7 Suutala N, Takalo T, Moisio T. Ferritic-austenitic solidification mode in austenitic stainless welds. Metalurgical Transactions A. 1980; I IA:717-725.

8 Wolynec S. Apostila do curso PMT-592. São Paulo: Escola Politécnica USP; 1994. Corrosão e proteção; p. 4.8-4.9.

9 Delong WT. A modified phase diagram for stainless steel weld metals. Metal Progress. 1960;77(2):99- I00B.

I0 Hammar O, Svensson UIN. A guide of solidification. Stockholm: Jernkontoret; 1977. 269 p.

I I Suutala N, Moisio T. Solidification and casting metals. London: The Metals Society; I979. Use of chromium and nickel equivalents in considering solidification mode in austenitic stainless steel welds; p. 3I0-3|4.

I 2 Suutala N, Takalo T, Moisio T. The relationship between solidification and microstructure in austenitic-ferritic stainless steel welds. Metallurgical Transactions A: Physical Metallurgy and Materials Science. 1979; I0A:5 I 2-5 I4.

I 3 Takalo T, Suutala N, Moisio T. Austenitic solidification mode in austenitic stainless steel welds. Metallurgical Transactions A: Physical Metallurgy and Materials Science. 1979; I0A: I I 73-I I8I.

I4 Suutala N, Takalo T, Moisio T. Single-phase ferritic solidification mode in austenitic-ferritic stainless steel welds. Metallurgical Transactions A: Physical Metallurgy and Materials Science. 1979; I0A: I I83-I 190. 
I5 Blanc G, Tricot R. Solidification, ségrégation et homogénéisation des aciers inoxydables austénitiques contant de la ferrite delta. Les Mémoires Scientifiques de la Revue de Métallurgie. 197।; I 1:735-753.

16 Fredriksson H. Solidification and casting metals. London: The Metals Society; 1979. Transiction from peritetic to eutectic reaction in iron-base alloys; p. 131-138.

17 Pryce L, Andrews KW. Practical estimation of composition balance and ferrite content in stainless steels. Journal of the Iron and Steel Institute. 1960;195:4I5-4I7.

18 Guiraldenq P. Action alphagéne et gammagéne des principaux elements d'addition dans le aciers inoxydables nickelchrome derives du type 18-10. Les Mémoires Scientifiques de la Revue de Métallurgie. 1967; 1 :907-939.

19 Hull FC. Delta ferrite and martensite formation in stainless steels. Welding Research Supplement. 1973;52(5):193s203 s.

20 Wrigth, I. G. Metals handbook. 9th ed. v. 13. Ohio: ASM; 2003. High-temperature corrosion; p. 97-103.

2I Kane RD. Metals handbook. v. I3A. Ohio: ASM; 2003. Corrosion: fundamentals, testing, and protection - hightemperature gaseous corrosion; p. 228-235.

22 Wasielewski GE, Rapp RA. The superalloys, vital high temperature gas turbine materials for aerospace and industrial power. New Jersey: John Wiley \& Sons Inc; 1972. High-temperature oxidation; p. 287-316.

23 American Society for Testing and Materials. ASTM E562-02: standard test method for determining volume fraction by systematic manual point count. West Conshohocken: ASTM; 2002.

24 American Society for Testing and Materials. ASTM E 1086-08: standard test method for optical emission vacuum spectrometric analysis of stainless steel by the point-to-plane excitation technique, West Conshohocken: ASTM; 2008.

Recebido em: 7 Nov. 2016

Aceito em: 9 Fev. 2017 